\title{
Contribuições da Tecnologia para Assistência de Enfermagem da Unidade de Terapia Intensiva
}

\author{
Vinícius Rodrigues Barboza Siqueira \\ Bacharel em Enfermagem pelo Centro Universitário do Vale do Ipojuca (UNIFAVIP|DeVry). \\ Pós-graduando em Enfermagem do Trabalho pela Faculdade Venda Nova do Imigrante (FAVENI) \\ 凶vinicius-rodrigues-49@hotmail.com \\ Wendyza Priscila de Carvalho Vasconcelos \\ Bacharel em Enfermagem pelo Centro Universitário do Vale do Ipojuca (UNIFAVIP|DeVry). \\ Pós-graduanda em Enfermagem Obstétrica pelo Centro de Ensino, formação, aperfeiçoamento profissional e pesquisa (CEFAPP) \\ 凶endyzapriscyla@hotmail.com \\ Gleidson Monteiro dos Santos \\ (In memorian) \\ Enfermeiro pelo Centro Universitário do Vale do Ipojuca (UNIFAVIP/DeVry). \\ Especialista em urgência e emergência pelo Centro de Ensino, formação, aperfeiçoamento profissional e pesquisa (CEFAPP). Especialista \\ em Terapia Intensiva. Especialista em Cardiologia e Hemodinâmica. . Mestre em Educação em Ciências e Matemática pela Universidade \\ Federal de Pernambuco (UFPE - CAA) \\ Gésica Kelly da Silva Oliveira \\ Enfermeira pelo Centro Universitário do Vale do Ipojuca (UNIFAVIP/DeVry). \\ Especialista em urgência e emergência pelo Centro de Ensino, formação, aperfeiçoamento profissional e pesquisa (CEFAPP). Espe cialista \\ em Ginecologia e Obstetrícia. Residente em Enfermagem Cirúrgica do Hospital das Clínicas da UFPE. Mestre em Educação em Ciências e \\ Matemática pela Universidade Federal de Pernambuco (UFPE - CAA) \\ $\bowtie$ gesicakelly.oliveira@gmail.com
}

Recebido em 10 de fevereiro de 2019

Aceito em 6 de junho de 2019

\section{Resumo:}

O processo tecnológico foi inserido em nosso cotidiano em todos os aspectos. A cada dia são implementados em nossa rotina novas tendências com o intuito de contribuir para a otimização do tempo. Diante disso houve o aprimoramento das práticas em saúde visando a evolução clínica positiva do paciente, oportunizando ao profissional a chance de agregar conhecimento e acompanhar as inovações no que concernem as "novas tecnologias" em prol das comunidades. Esta pesquisa objetivou investigar quais as contribuições da evolução tecnológica sobre a ótica dos enfermeiros dentro da Unidade de Terapia Intensiva (UTI), analisando as vantagens e desvantagens do uso da mesma na assistência ao paciente. Trata-se de um estudo transversal descritivo, de abordagem quantitativa, realizado com 14 enfermeiros do setor da UTI de um Hospital do interior de Pernambuco. Os resultados apontaram que a maioria dos equipamentos se encontrava em bom estado de conservação, além disso, os participantes pontuaram que o déficit do manuseio dos equipamentos, bem como a manutenção destes, se caracteriza como fatores que dificultam a assistência junto aos aparatos tecnológicos e equipamentos mais significativos dentro da Unidade de Terapia Intensiva. Os aparatos tecnológicos contribuem expressivamente para a melhoria da assistência à saúde, cabe a gestão condicionar fatores que propiciem o uso de equipamentos e aos profissionais que desenvolvem a assistência a capacidade e autonomia de utilizá-los de forma coerente.

Palavras-chave: Unidades de terapia intensiva, Enfermagem, Tecnologia em saúde. 


\title{
Contributions of the Technology for Nursing Assistance of the Intensive Care Unit
}

\begin{abstract}
:
The technological process was inserted in our daily life in every aspect, every day new trends are implemented in our routine with the intention of contributing to the optimization of the time, in front of this there was the improvement of the health practices aiming at the positive clinical evolution of the patient, giving the professional the chance to add knowledge and follow the innovations regarding "new technologies" for the benefit of the communities. This research aimed to investigate the contributions of technological evolution to the perspective of nurses within the Intensive Care Unit (ICU), analyzing the advantages and disadvantages of its use in patient care. This is a cross-sectional, descriptive, quantitative study conducted with 14 nurses from the ICU sector of a Hospital in the interior of Pernambuco. The results showed that most of the equipment was in good condition, in addition, the participants pointed out that the deficiency of the handling of the equipment, as well as the maintenance of these equipments, are characterized as factors that make it difficult to assist with the technological apparatuses and equipment more significant within the Intensive Care Unit.T he technological devices contribute expressively to the improvement of health care, it is up to the management to condition factors that allow the use of equipment and the professionals that develop the assistance the capacity and autonomy to use them in a coherent way.
\end{abstract}

Keywords: Intensive care units, Nursing, Technology in health.

\section{Contribuciones de la Tecnología para Asistencia de Enfermería de la Unidad de Terapia Intensiva}

\section{Resumen:}

El proceso tecnológico fue insertado en nuestro cotidiano en todos los aspectos, cada día se implementan en nuestra rutina nuevas tendencias con el fin de contribuir a la optimización del tiempo, ante eso hubo el perfeccionamiento de las prácticas en salud visando la evolución clínica positiva del paciente, oportunizando al profesional la oportunidad de agregar conocimiento y acompañar las innovaciones en lo que conciernen a las "nuevas tecnologías" en favor de las comunidades. Esta investigación objetivó investigar cuáles las contribuciones de la evolución tecnológica sobre la óptica de los enfermeros dentro de la Unidad de Terapia Intensiva (UTI), analizando las ventajas y desventajas del uso de la misma en la asistencia al paciente. Se trata de un estudio transversal descriptivo, de abordaje cuantitativo, realizado con 14 enfermeros del sector de la UTI de un Hospital del interior de Pernambuco. Los resultados apuntaron que la mayoría de los equipos se encontraban en buen estado de conservación, además, los participantes puntualizó que el déficit del manejo de los equipos, así como el mantenimiento de éstos, se caracteriza como factores que dificultan la asistencia junto a los aparatos tecnológicos y equipamientos más significativos dentro de la Unidad de Terapia Intensiva. Los aparatos tecnológicos contribuyen expresivamente a la mejora de la asistencia a la salud, cabe la gestión condicionar factores que propicien el uso de equipos y los profesionales que desarrollan la asistencia a la capacidad y autonomía de utilizarlos de forma coherente.

Palabras clave: Unidades de terapia intensiva, Enfermería, Tecnología en salud. 


\section{INTRODUÇÃo}

O processo tecnológico está implantado em nosso cotidiano em todos os aspectos, tanto no âmbito pessoal quanto profissional, rotineiramente são inseridos em nosso cotidiano novas tendências com o intuito de contribuir principalmente para a otimização do tempo, gerando mudanças significantes (OLIVEIRA; SOUZA, 2014).

Diante desse sistema de evolução tecnológica, houve o aprimoramento das práticas em saúde, que por sua vez, foram e são incrementadas visando à evolução clínica positiva dos pacientes, oportunizando ao profissional agregar conhecimento e acompanhar as inovações no que concernem às "novas tecnologias" em prol das comunidades (FERNANDES et al., 2010).

Diante dos grandes avanços tecnológicos na saúde, observou-se que a tecnologia está subdividida e identificada em três eixos: dura, leve-dura e leve, compreendendo que as "duras" estão relacionadas aos equipamentos, máquinas ou instrumentais utilizados, as "leve-duras" são os saberes estruturados e associados a equipamentos tecnológicos (tecnologia dura), e as "leves" compreendem a relação entre o profissional e o usuário, numa assistência diretamente ligada a aplicação dos procedimentos teórico-práticos (ABREU; AMENDOLA; TROVO, 2017).

Segundo Fernandes (2010), as técnicas que antes demandavam mais trabalho e recursos humanos, hoje são realizadas por equipamentos das mais variadas formas e tamanhos, mostrando que além do aprimoramento de procedimentos do passado, a tecnologia também propicia a efetiva ascensão de práticas que vislumbram sempre a convalescença dos usuários.

Logo, há a necessidade de um suporte às equipes visando a excelência no atendimento, com propriedade e qualidade na atuação do serviço ofertado no setor, ressalta-se a importância de que alguns elementos devem ser trabalhados com as equipes a fim de atingir os objetivos esperados, a qualificação desses profissionais frente aos novos equipamentos, a assistência técnica especializada e a manutenção dos aparelhos são indispensáveis, elevando a qualidade da assistência prestada e prevenindo a ocorrência de possíveis acidentes (PEREZ et al., 2014). 


\section{Siqueira et al.}

Nesse contexto, o surgimento e a estruturação das Unidades de Terapia Intensiva (UTI) nos hospitais ocorreu, principalmente, para acolher os pacientes em estado crítico com prognóstico positivo, mas também, considerando a essa percepção da necessidade de acompanhar os processos tecnológicos contemporâneos que evoluem em favor da assistência em saúde, de modo a ofertar um atendimento diferenciado, vigilância constante da equipe multiprofissional que perdure às 24 horas do dia (MENDES, 2010).

De acordo com o Ministério da Saúde (2010) a Unidade de Terapia Intensiva (UTI) é uma área crítica com pacientes graves, que requerem o máximo de atenção e assistência profissional especializada em tempo integral e contínua, materiais específicos e recursos necessários ao diagnóstico (monitorização e terapias exclusivas do setor), havendo sempre a necessidade de investimento em educação continuada para todos os profissionais que ali trabalham, visando assim, a segurança e a proteção ao paciente.

Tendo em vista a necessidade de cuidados mais complexos exigidos no setor, a presença do enfermeiro se torna indispensável, considerando sua responsabilidade no exercício da enfermagem, que dentre tantas atribuições se propõe a viabilizar os subsídios necessários nas áreas de gestão, vigilância e assistência qualificada aos pacientes, evidenciando a essência de uma rotina organizada e dinâmica para a realização de um trabalho isento de danos e/ou de riscos (MENDES, 2010).

Com as mudanças trazidas pelo avanço tecnológico os profissionais de enfermagem foram condicionados a passarem por um processo de adaptação de modo a gerar uma harmonia entre a teoria, os procedimentos técnicos e a tecnologia, tendo em vista que com os avanços tecnológicos surgem um leque de benefícios ao paciente que perpassa pela redução do tempo de seu tratamento/internação ou em alguns casos assumem a manutenção da vida (SALVADOR et al., 2012).

Ressalta-se, que o processo evolutivo que os cuidados de enfermagem sofreram sob a influência direta da implementação e desenvolvimento tecnológico na UTI, possibilitou o aprimoramento do cuidado com os enfermos potencializando a fidedignidade de dados obtidos em seu acompanhamento e otimizando o tratamento, por consequência, diminuindo seu tempo de internação.

Desta forma este estudo objetivou analisar as contribuições trazidas pelos avanços relacionados à tecnologia sobre a ótica dos enfermeiros, evidenciando a relação entre a 
qualidade da assistência prestada por toda equipe multidisciplinar e a inserção das tecnologias no setor de alta complexidade.

\section{METODOLOGIA}

A presente pesquisa tratou-se de um estudo transversal descritivo, de abordagem quantitativa. Segundo Bertucci (2009) o estudo descritivo visa descrever as características de determinada população ou fenômeno, caracterizando o levantamento de opiniões, atitudes e crenças, do grupo estudado.

A pesquisa foi realizada na Unidade de Terapia Intensiva do Hospital Regional do Agreste Dr. Waldemiro Ferreira (HRA), considerada uma Unidade de Terapia Intensiva tipo A (UTI-A), direcionada ao público adulto segundo a RDC № 7, de 24 de fevereiro de 2010, está unidade hospitalar pertence a rede estadual de saúde, o mesmo é considerado referência em trauma de média e alta complexidade.

A escolha desse local para a realização do estudo foi dada pela área apresentar maior densidade tecnológica, que irão ser abordados ao longo desse trabalho. Além disso, o setor de UTI se torna parte imprescindível na assistência devido ser o ambiente para onde são direcionados os pacientes graves, mas potencialmente recuperáveis, é caracterizado por ser um espaço isolado, silencioso, e onde está disposto a maioria dos equipamentos que são destinados a manutenção da vida dos pacientes críticos, desta forma o setor promoveu as condições necessárias para conduzir a pesquisa da forma desejada.

A amostra deste estudo foi composta por todos os 14 enfermeiros plantonistas atuantes na UTI do hospital supracitado, constituindo uma amostra censitária. Foram incluídos no estudo todos os enfermeiros plantonistas que se encontravam em efetivo exercício e aceitaram participar da pesquisa por meio da assinatura do termo de consentimento livre esclarecido (TCLE). Excluiu-se aqueles que porventura estavam sob atestado, licenças, ou não foram encontrados.

A pesquisa foi autorizada pela Secretaria Estadual de Saúde, além disso, foi submetida a plataforma Brasil e aprovada pelo Comitê de Ética em Pesquisa (CEP) do Centro 


\section{Siqueira et al.}

Universitário do Vale do Ipojuca, obtendo parecer favorável, de número: 1.878.945, em decorrência das questões éticas que permeiam as pesquisas que envolvem seres humanos, atendendo a resolução de nº 466 de 2012 do Conselho Nacional de Saúde.

A coleta dos dados foi realizada no período de fevereiro a março de 2017, por meio de questionário autoaplicável composto por 12 perguntas de múltipla escolha, no intuito de atender os interesses deste estudo.

Após coleta dos dados os mesmos foram digitados e processados no programa Microsoft Excel versão 2013, em busca de erros e omissões, após a análise dos resultados estes foram apresentados na forma tabular. A partir disto, os dados foram confrontados com base nos vários referenciais teóricos que amparam as interpretações.

\section{RESULTADOS E DISCUSSÃO}

O setor de UTI destaca-se quando se diz respeito a desenvolvimento tecnológico, devido à necessidade que a área apresenta de manter uma diversidade de materiais e equipamento, pois é para onde são destinados os pacientes considerados críticos.

A tabela 1 faz referência às características sócio demográficas da população em estudo, ordenada por sexo, faixa etária, formação, tempo de formação e experiência em atividades de enfermagem exercidas em setores de terapia intensiva, de modo a evidenciar as características da população da amostra e demonstrar suas especificações. 
Contribuições da Tecnologia para Assistência

de Enfermagem da Unidade de Terapia Intensiva

Tabela 1 - Características sociodemográficas dos participantes da pesquisa

\begin{tabular}{|c|c|c|}
\hline CARACTERÍSTICAS SOCIODEMOGRÁFICAS & $\begin{array}{l}\text { FREQUÊNCIA } \\
\text { "N" } \\
\end{array}$ & $\begin{array}{c}\text { PERCENTUAL } \\
\text { “\%" }\end{array}$ \\
\hline \multicolumn{3}{|l|}{ SEXO } \\
\hline Feminino & 13 & $92,9 \%$ \\
\hline Masculino & 01 & $7,1 \%$ \\
\hline Total & 14 & $100 \%$ \\
\hline \multicolumn{3}{|l|}{ FAIXA ETÁRIA (EM ANOS) } \\
\hline $20-25$ & 02 & $14,3 \%$ \\
\hline $26-30$ & 06 & $42,9 \%$ \\
\hline $31-40$ & 03 & $21,5 \%$ \\
\hline $41-45$ & 01 & $7,1 \%$ \\
\hline $46-50$ & 01 & $7,1 \%$ \\
\hline$>50$ & 01 & $7,1 \%$ \\
\hline Total & 14 & $100 \%$ \\
\hline \multicolumn{3}{|l|}{ FORMAÇÃo } \\
\hline Graduação & 04 & $28,6 \%$ \\
\hline Especialização & 09 & $64,3 \%$ \\
\hline Mestrado & 01 & $7,1 \%$ \\
\hline Doutorado & 00 & $0 \%$ \\
\hline Total & 14 & $100 \%$ \\
\hline \multicolumn{3}{|l|}{ TEMPO DE ATUAÇÃO NA ENFERMAGEM } \\
\hline 01-03 anos & 04 & $28,6 \%$ \\
\hline 04-06 anos & 05 & $35,7 \%$ \\
\hline 07-10 anos & 00 & $0 \%$ \\
\hline$>11$ anos & 05 & $35,7 \%$ \\
\hline Total & 14 & $100 \%$ \\
\hline \multicolumn{3}{|l|}{ TEMPO DE ATUAÇÃO DA UTI } \\
\hline 00-02 anos & 07 & $42,9 \%$ \\
\hline 03-05 anos & 01 & $7,1 \%$ \\
\hline 06-10 anos & 02 & $14,3 \%$ \\
\hline$\geq 10$ anos & 05 & $35,7 \%$ \\
\hline Total & 14 & $100 \%$ \\
\hline
\end{tabular}

Fonte: Os autores; Caruaru, 2018. 


\section{Siqueira et al.}

Dos 14 enfermeiros entrevistados, $92,8 \%$ eram do sexo feminino e $7,1 \%$ do sexo masculino, a proporcionalidade encontrada foi de 1 homens para 13 mulheres, demonstrando a prevalência histórica do sexo feminino no campo da enfermagem (SOUZA, 2014). Sobre a faixa-etária, $42,9 \%$ da amostra apresentavam entre 26 a 30 anos de idade o que nos aponta uma predominância de enfermeiros jovens.

Em relação à formação 64,3\% afirmaram ter especialização em Unidade de Terapia Intensiva (UTI), enquanto $28,6 \%$ apresentavam apenas a graduação e apenas 7,1\% relataram a realização de mestrado.

No que concerne ao tempo de atuação de enfermagem destacou-se que 35,7\% dos profissionais atuavam entre quatro entre 4-6 anos correspondendo também em mesma porcentagem de $35,7 \%$ os profissionais que atuavam a mais de 11 anos. Do total de pesquisados foi encontrado que $42,9 \%$ apresentam pelo menos dois anos de atuação no setor de UTI, destacando que possuem uma experiência profissional considerável nesse local.

Percebemos que a maioria dos entrevistados faz ou fizeram especializações relacionadas à Terapia Intensiva, isso potencializa o conhecimento dos profissionais da área, facilitando o domínio das informações especificas do setor de atuação, de modo que as especializações supracitadas quando associadas a uma educação continuada trazem inúmeros benefícios ao desempenho profissional diante das tecnologias (LAZZARI; SHIMIDT; JUNG, 2012).

O que enfatiza a ideia de que o processo de qualificação e atualização é indispensável para assegurar a qualidade da assistência na área de saúde, particularmente nas ações de cunho complexo requerendo disseminação de conhecimentos e informações que são utilizados para o aperfeiçoamento constante do atendimento em saúde e na preservação da qualidade da assistência prestada aos pacientes (LAZZARI; SHIMIDT; JUNG, 2012).

A tabela 2 relaciona as variáveis: estado de conservação dos equipamentos utilizados na UTI, fatores que dificultam a assistência junto aos aparatos tecnológicos, equipamentos mais significativos para assistência de enfermagem no setor de UTI frente ao discernimento do profissional enfermeiro. 
Tabela 2 - Percepção dos enfermeiros frente às vantagens e desvantagens encontradas com a utilização das tecnologias na UTI.

\begin{tabular}{lcc}
\hline \multicolumn{1}{c}{ UTILIZAÇÃo DA TECNOLOGIA NA UTI } & $\begin{array}{c}\text { FREQUÊNCIA } \\
\text { "N" }\end{array}$ & $\begin{array}{c}\text { PERCENTUAL } \\
\text { “\%" }\end{array}$ \\
\hline $\begin{array}{l}\text { ESTADO DE CONSERVAÇÃo DOS EQUIPAMENTOS UTILIZADOS } \\
\text { NA UTI }\end{array}$ & & \\
Em bom estado de conservação & 12 & $85,8 \%$ \\
Em ótima condição de uso & 01 & $7,1 \%$ \\
Em estado precário de conservação & 01 & $7,1 \%$ \\
Sem condições de uso & 00 & $0 \%$ \\
Total & 14 & $100 \%$
\end{tabular}

FATORES QUE DIFICULTAM A ASSISTÊNCIA JUNTO AOS

\section{APARATOS TECNOLÓGICOS}

Déficit para manusear os equipamentos.

06

06

02

14

*EQUIPAMENTOS MAIS SIGNIFICATIVOS PARA

ASSISTÊNCIA DE ENFERMAGEM NO SETOR DE UTI

Cardioversor/ desfibrilador

14

Monitor multiparamétrico

Ventilador mecânico

Bomba de infusão venosa

Oxímetro de pulso

Aparelho de glicemia capilar
$42,8 \%$

$42,8 \%$

$14,4 \%$

$100 \%$

Fonte: Os autores; Caruaru, 2018

* Respostas múltiplas de Enfermeiros participantes da pesquisa.

Diante da diversidade de equipamentos existentes no setor de UTI, destacaram-se seis desses equipamentos, devido a sua capacidade de mensurar parâmetros essências para uma avalição mais fidedigna do quadro clínico geral do paciente. Dentre os resultados obtidos sobre tais equipamentos privativos do setor UTI que se destacam como 
fundamentais, $100 \%$ dos pesquisados afirmaram que seriam o desfibrilador, o monitor multiparâmentros e o ventilador mecânico.

Com tais aparatos presentes na unidade se torna evidente o quanto eles influenciam de forma positiva e imediata no quadro clínico dos pacientes, quando associados com a ação dos profissionais ao manusear e interpretar os dados obtidos de forma clara e objetiva, estimulam a melhora no da qualidade de vida dos mesmos (SILVA et al., 2015).

Destacou-se também o aparelho de glicemia capilar com $71,4 \%$, os entrevistados o consideraram relevante na assistência, enfatizando que o mesmo tem função considerável na avalição e acompanhamento de distúrbios relacionados à glicemia, que quando alterada pode vir a agravar doenças preexistentes dos pacientes (PEREZ et al., 2014).

Todos os equipamentos supracitados tem sua importância na assistência prestada ao usuário, em conjunto, têm a capacidade de expor e direcionar a problemática do cliente, levando a uma ação mais efetiva e precisa de toda a equipe em relação ao combate dos distúrbios e agravos encontrados (SALVADOR et al., 2012).

Contudo, observa-se que $64,3 \%$ dos enfermeiros alegam que após a inserção de equipamentos com alto teor tecnologico na UTI, desencadeou-se um processo de mecanização da assistência prestada aos pacientes, o que pode estar relacionado a inúmeros fatores como sobrecarrega do responsável pelo planejamento e organização dos processos que uni as tecnologias e domínio técnico (CARGNIN et al., 2016).

Outro ponto sinalizado é a autonomia que os equipamentos encontrados no setor apresentam, o que por sua vez pode afetar o processo do cuidar tornando-o automatizado, estas questões quando identificadas, devem ser corrigidas de forma emergencial visando diminuir ou erradicar as possíveis maleficências trazidas pela má utilização dos aparelhos (CARGNIN et al., 2016).

Ao indagar sobre o estado de conservação dos equipamentos utilizados nota-se que $85,8 \%$ dos participantes deste estudo, relataram que os aparelhos que estão em uso frequente encontram-se em bom estado de conservação, contraposto a isso $42.8 \%$ relataram que entre as dificuldades encontradas na assistência ao paciente sob o uso das tecnologias foi o déficit na manutenção dos equipamentos considerados sobressalentes, dispostos em áreas de armazenamento de material, evidenciando que mesmo os aparatos estando presentes na unidade hospitalar e em sua maioria em bom estado de conservação, existem 
equipamentos que estão em desuso devido à falta de manutenção, afetando diretamente a prestação plena dos serviços prestados aos pacientes (PEREZ et al., 2014).

A dificuldade em manusear os equipamentos do setor também ganhou destaque quando citada por $42,8 \%$ dos participantes, mesmo que os profissionais atuantes possuam algum tipo de especialização na área, foi evidenciada, talvez, uma possível ausência de educação continuada, necessitando que haja investimento nesse sentido.

Quanto à interação da implementação da tecnologia que permite apoiar a assistência de enfermagem frente aos cuidados com os pacientes, teve-se como resultado que a tecnologia exerce uma influência direta e positiva na assistência, elando as tendências tecnológicas com os procedimentos técnicos da equipe, o que vislumbra uma assistência ampla e qualificada para com o usuário (GOMES et al., 2017).

Sobre o processo do cuidado exercido pela equipe de saúde, 100\% dos participantes evidenciaram a necessidade de se basear em um cuidado holístico aliando as tecnologias inseridas no setor com o conhecimento teórico prático do profissional, estes cuidados devem sempre estar em harmonia com o binômio maquina-profissional, mantendo a frente os princípios éticos e morais (ALMEIDA; FóFANO, 2016).

Fica claro que a tecnologia acrescenta, em particular, na assistência de enfermagem ofertada no setor de terapia intensiva, todavia, ainda se faz necessário a implementação de rotinas administrativas no que diz respeito a manutenção dos equipamentos e sobre a educação continuada para a equipe no que se refere ao manuseio destes (SILVA et al., 2015).

\section{CONSIDERAÇÕES FINAIS}

Diante dos resultados obtidos tornou-se evidente a transformação e a evolução da assistência com a implementação de equipamentos de alto teor tecnológico dentro da Unidade de Terapia Intensiva (UTI), isso emergiu com as inúmeras alterações ocorridas na dinâmica da assistência após a associação das abordagens teórico-práticas e dos aparatos tecnológicos. A gama tecnológica por sua vez, se renova e cresce a cada ano tornando os equipamentos ainda mais rápidos e precisos em suas destinas funções com intuito de aperfeiçoar e tornar mais eficaz o processo de cuidar. Nesse sentido, podemos perceber o 
quanto esse avanço repercutiu diretamente na assistência prestada ao paciente crítico influenciando nos padrões de diagnósticos e tratamentos.

Podemos concluir que o a inserção de equipamentos de alto teor tecnológico auxilia e beneficia as intervenções da equipe de enfermagem no âmbito da UTI, contribuindo de forma direta para a existência de um tratamento mais efetivo e pleno, além é claro de promover maior segurança nos cuidados prestados e fidedignidade nos resultados, desta forma podendo também minimizar -o tempo de internação na unidade e otimizar a recuperação dos usuários nos serviços de saúde.

Os profissionais de enfermagem que atuam na UTI devem utilizar a os equipamentos de alto teor tecnológico de forma consciente e não deixar seus procedimentos "robotizados" utilizando sempre de seus conhecimentos técnico-científicos, contemplando os indivíduos em sua integralidade e dignidade enquanto ser humano. Neste sentido, incumbe aos profissionais, o pensamento crítico e reflexivo, na utilização dos equipamentos disponíveis, na perspectiva de oferecer uma assistência por excelência, por meio da articulação entre ciência, habilidade e tecnologia.

\section{REFERÊNCIAS}

ABREU, T. F. K; AMENDOLA, F; TROVO, M. M. Tecnologias relacionais como instrumentos para o cuidado na Estratégia Saúde da Família. Revista Brasileira de Enfermagem, Brasília , v. 70, n. 5, p. 981-987, out. 2017. Disponível em <http://www.scielo.br/scielo.php?script=sci_arttext\&pid=S003471672017000500981\&lng=pt\&nrm=iso>. Acessos em: 09 fev. 2018.

ALMEIDA, Q.; FÓFANO, G.A. Tecnologias leves aplicadas ao cuidado de enfermagem na unidade de terapia intensiva: uma revisão de literatura. HU Revista. Juiz de Fora. v. 42, n. 3, p. 191-196, 2016. Disponível em < http://ojs2.ufjf.emnuvens.com.br/hurevista/article/view/2494 > Acesso em: 09.06.2017.

BERTUCCI, J.L.O. Metodologia básica para elaboração de trabalhos de conclusão de cursos (TCC). 1.ed. São Paulo: Atlas, 2009.

BRASIL. Ministério da Saúde. Agência Nacional de Vigilância Sanitária. RESOLUÇÃO- № 7, DE 24 DE FEVEREIRO DE 2010. Dispõe sobre os requisitos mínimos para funcionamento de Unidades de Terapia Intensiva e dá outras providências. 2010. Disponível em <

http://bvsms.saude.gov.br/bvs/saudelegis/anvisa/2010/res0007_24_02_2010.html> Acesso em: 07.06.2017.

CARGNIN, M.C.S; OTTOBELLI, C; BARLEM, E.L.D; CEZAR-VAS, M. R. Tecnologia no cuidado da enfermagem e a carga de trabalho em UTI. Revista de enfermagem UFPE on line. Recife. v.10, n. 2, p. 903-7, 2016. Disponível em <https://periodicos.ufpe.br/revistas/revistaenfermagem/article/viewFile/11035/12431 > Acesso em: 06.09.2017.

FERNANDES, G.T; ALVES, L.N; CUNHA, L.S; MONTEIRO, M.C; BATISTA, R.S. Tecnologia de Ponta em Unidade de Terapia Intensiva (UTI) e sua Influência na Humanização do Cuidado de Enfermagem. Rio de Janeiro; 
s.n; 2010. Disponível em < http://bvsms.saude.gov.br/bvs/publicacoes/hfb/roberto_batista.pdf> Acesso em: 06.08.2017.

GOMES, A.T.L; ASSIS, I. M. S; FERREIRA, L. L; BEZERRIL, M.S; CHIAVONE, F. B.T; DSNTOD, V. E. P. Tecnologias aplicadas à segurança do paciente: uma revisão bibliométrica. Revista de Enfermagem do Centro-Oeste Mineiro, v.7, p.1-11, 2017. Disponível em < http://seer.ufsj.edu.br/index.php/recom/article/viewFile/1473/1719 > Acesso em: 26.07.2018.

LAZZARI, D.D.; SHIMIDT, N.; JUNG, W. Educação continuada em Unidade de Terapia Intensiva na percepção de enfermeiras. Revista de Enfermagem da UFSM. v.2, n.1, p 88-96, 2012. Disponível em < https://periodicos.ufsm.br/reufsm/article/view/4592> Acesso em: 15.08.2017.

MENDES, T.N.C. UTI - Passado, Presente e Futuro. Trabalho de conclusão de curso (Graduação) - Universidade Estadual do Maranhão, Centro de Estudos Superiores de Itapecuru-mirim CESIM, Itapecuru-mirim, 2010.

OLIVEIRA, E.B.; SOUZA, N.V.M. Estresse e inovação tecnológica em unidade de terapia intensiva de cardiologia: tecnologia dura. Revista Enfermagem UERJ. Rio de Janeiro. v.20, n.4, p 457-62, 2012. Disponível em < https://www.e-publicacoes.uerj.br/index.php/enfermagemuerj/article/view/4768 > Acesso em: 01.05.2017.

PEREZ JÚNIOR, E.F; OLIVEIRA, E.B; SOUZA, N. V. D. O; LISBOA, M.T. L; SILVINO, Z. R. Segurança no desempenho e minimização de riscos em terapia intensiva: tecnologias duras. Revista Enfermagem UERJ, Rio de Janeiro. v. 22, n. 3, p. 327-33, 2014. Disponível em < http://www.facenf.uerj.br/v22n3/v22n3a06.pdf > Acesso em: 01.05.2017.

SALVADOR, P.T.C.O; OLIVEIRA, R. K .M; COSTA, T. D; SANTOS, V. E. P; TOURINHO, F. S. V. Tecnologia e inovação para o cuidado em enfermagem. Revista Enfermagem UERJ. Rio de Janeiro. v.20, n.1, p 111-7, 2012. Disponível em < http://www.facenf.uerj.br/v20n1/v20n1a19.pdf > Acesso em 18.05.2017.

SILVA, R.C; FERREIRA, M.A; APOSTOLIDIS, T; BRANDÃO, M.A.G. Um marco conceitual da clínica do cuidado de enfermagem na terapia intensiva. Revista Latino-Americana de Enfermagem . v. 23, n. 5, p. 837-45, 2015. Disponível em < http://www.scielo.br/pdf/rlae/v23n5/pt_0104-1169-rlae-23-05-00837.pdf> Acesso em: 20. 06.2017.

SOUZA, L.L. Representações de gênero na prática de enfermagem na perspectiva de estudantes. Ciências \& Cognição, v. 19, n.2, p. 218-232, 2014. Disponível em<http://webcache.googleusercontent.com/search?q=cache:a75ipwr9_7gJ:cienciasecognicao.org/revista/in dex.php/cec/article/download/908/pdf_13+\&cd=1\&hl=pt-BR\&ct=clnk\&gl=br> Acesso em 12.08.2017.

\section{(cc) Br}

Este trabalho está licenciado com uma Licença Creative Commons - Atribuição 4.0 Internacional. 